\section{Dor Orofacial: Compreendendo o Processo Pelos Pontos de Vista do Paciente e do Cuidador}

\section{Orofacial Pain: Understanding the Process from the Patient and the Caregiver's Standpoint}

\section{RESUMO}

Uma situação bastante comum relatada por pacientes que procuram por tratamento clínico é a presença de sintomatologia dolorosa. A dor é uma experiência pessoal e subjetiva influenciada pela experiência cultural, pelo conhecimento da situação, atenção e outras variáveis psicológicas. Este trabalho teve como objetivo proporcionar ao estudante da área de saúde, aos docentes e aos pacientes, por meio de entrevistas e aplicação da ferramenta McGill Pain Questionnaire, melhor conhecimento sobre os mecanismos desencadeantes das dores temporomandibulares (DTMs) e sobre a eficiência do uso de placa miorrelaxante. A melhor relação clínico/cliente na discussão dos sinais, sintomas e intensidade de dor referida, bem como a motivação dos cuidadores para o melhor acolhimento e atendimento humanizado dos clientes portadores de DTMs crônicas, vislumbrando ação positiva no ensino, na pesquisa e na extensão com atendimento de melhor qualidade foram incentivadas. Após três anos de acompanhamento clínico de 39 pacientes, com avaliação da dor qualitativamente, pode-se concluir que as expectativas dos clientes e cuidadores com relação ao tratamento clínico das DTMs nem sempre são atendidas em igual importância, apesar de o tratamento clínico com a placa ter sido satisfatório. Notou-se ainda a falta de adesão ao tratamento e seguimento de caso após o cessamento da dor.

Palavras-chave: Placa miorrelaxante. Questionário de avaliação da dor. Qualidade de vida.

\section{ABSTRACT}

A common situation reported by patients seeking clinical treatment is the presence of pain. Pain is a subjective and personal experience influenced by cultural experience, knowledge of the situation, attention and other psychological variables. This study aimed
Bárbara Graziele Ramos, Samira Falleiros Ortiz, TAMIRIS BITTENCOURT FONSECA E VINICIUS Pedrazz I 
to provide to the student health care, teachers and patients, through interviews and the McGill Pain Questionnaire tool, better understanding of the mechanisms triggering the temporomandibular pain (TMP), also the efficiency of occlusal splint use. The best clini-

$\mathrm{cal} /$ client relationship by means of discussion on the signs, symptoms and intensity of pain reported, as well as the motivation of caregivers to better care and humanized assistance TMP chronic customer, seeing positive action in teaching, research and extension better quality care were encouraged. After three years of clinical follow-up of 39 patients with pain assessment qualitatively, it can be concluded that the expectations of clients and caregivers regarding the clinical treatment of TMD are not always met in equal importance, although the clinical treatment with plaque being satisfactory. It was noted also the lack of adherence to treatment and follow-up case, after pain ceasing.

Keywords: Occlusal splints; McGill Pain Questionnaire; Quality of Life.

\section{INTRODUÇÃO}

Uma situação bastante comum relatada por pacientes que procuram por tratamento clínico é a presença de sintomatologia dolorosa. A dor é uma experiência pessoal e subjetiva influenciada pela experiência cultural, pelo conhecimento da situação, atenção e outras variáveis psicológicas. $O$ processo de dor não é simplesmente a captação e transmissão de estímulos nociceptivos e a percepção destes por centros nervosos superiores. Agressões ou doenças podem produzir sinais neurais que entram em um sistema nervoso ativo, o qual é o substrato de experiências passadas, cultura e estado emocional $[1,2]$.

Um paciente que é diagnosticado como portador de disfunção temporomandibular (DTM) pode ser considerado como um grande desafio clínico. A etiologia dessas desordens nem sempre é conhecida, sendo muitas vezes de origem multifatorial, dificultando o diagnóstico e o estabelecimento de uma terapia adequada para cada paciente. Todos estes fatores têm levado ao conceito de que nosso objetivo clínico não é o tratamento em si, mas sim a sua administração. De fato, a administração ou condução dos pacientes que sofrem de desordens temporomandibulares, pode envolver uma série de abordagens terapêuticas, incluindo a farmacoterapia [2].

A opção pelo uso do McGill Pain Questionnaire deve-se ao fato de este ter se mostrado um instrumento bastante útil na mensuração da dor. Sua versão traduzida e adaptada, além disso, validou-o para sua utilização como instrumento em português de mensuração da dor [5].

\section{MATERIAIS E MÉTODOS}

As atividades propostas e realizadas neste projeto foram:

1. Após consentimento livre e esclarecido do supervisor de clínicas e dos responsáveis pelas disciplinas clínicas da Faculdade de Odontologia de Ribeirão Preto da Universidade de São Paulo (FORP-USP), as alunas de graduação envolvidas no projeto, 
bolsistas do Programa Aprender com Cultura e Extensão (Projeto 2310) selecionaram os clientes que preencheram os requisitos para ingressar no estudo: portadores de DTMs crônicas, em tratamento nas clínicas da FORP sendo considerados de ambos os gêneros e com idade acima de dezesseis anos;

2. De posse das fichas clínicas, e após conhecimento detalhado dos casos, foram efetuadas entrevistas onde o cliente tinha toda liberdade de expressar seus problemas relacionados à dor, sendo investigada a história médica anterior, familiar, história dental, profissão, uso de medicamentos etc. Todos os clientes receberam e assinaram TCLE (termo de consentimento livre e esclarecido) aprovado pelo Comitê de Ética em Pesquisa da FORP-USP (Processo 2010.1.1130.58.9);

3. Foi então aplicada a versão em português do McGill Pain Questionnaire [5], para diferenciação entre dor somática, afetiva ou miscelânea, e uma Escala Visual Analógica (EVA) para mensuração da dor (Figura 1);

\section{McGill Pain Questionnaire - Português}

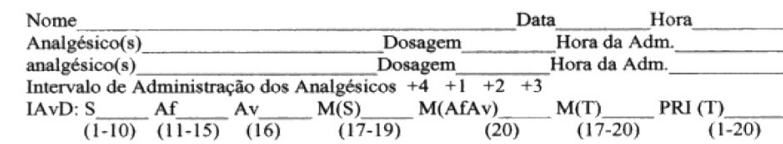

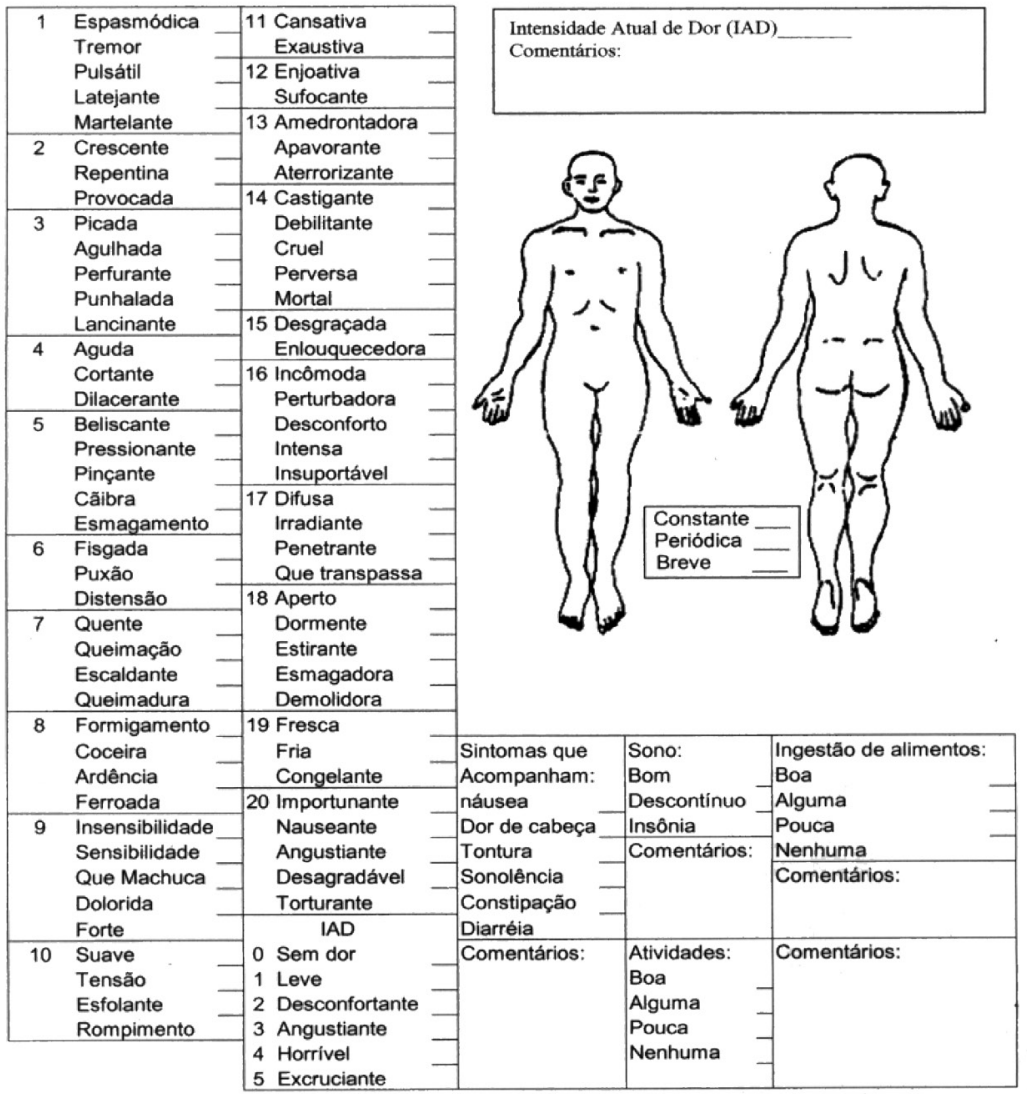

Figura 1 - McGill Pain Questionnaire - Versão em Língua Portuguesa. 
4. Os resultados após três anos de acompanhamento foram tabulados e discutidos primeiramente pelo coordenador do projeto e bolsistas envolvidas, depois, em uma segunda etapa, com os docentes clínicos onde o projeto foi desenvolvido, finalizando com uma discussão mais ampla;

5. Envolver alunos de graduação e buscar alternativas que possam melhorar a relação clínico/cliente e mais e melhor qualidade de tratamento para alívio ou supressão da dor.

\section{RESULTADOS}

As bolsistas do Programa Aprender com Cultura e Extensão conviveram com uma realidade ao mesmo tempo próxima a elas (a dor, a origem da dor, a quantificação da dor) e paradoxalmente distante (a qualidade da dor, o que o cliente espera de um serviço público de atendimento, no diagnóstico e alívio/supressão da dor). Quanto às bolsistas, uma era graduanda do curso de Odontologia da Faculdade de Odontologia de Ribeirão Preto, outra do curso de Fisioterapia e outra do curso de Fonoaudiologia da Faculdade de Medicina de Ribeirão Preto da USP. O relacionamento entre elas, e também entre elas e os docentes das disciplinas e os clientes do projeto foi incrementado com o tempo, demonstrando que a extensão tem papel fundamental na interlocução entre os agentes de saúde (como imaginamos ser também em outras áreas/domínios de ensino e pesquisa) e a sociedade. Nenhum tratamento terá o sucesso almejado se o cliente não for consultado e participativo das ações desenvolvidas.

$\mathrm{O}$ coordenador do projeto aprendeu muito com as bolsistas (inclusive nos momentos em que encontraram dificuldades durante a aplicação das ferramentas (McGill/ Escala EVA) e na busca de satisfação pessoal dos clientes) e com o "olhar humanitário" que as mesmas demonstraram quando estas ofereceram de suas próprias verves a oportunidade de manifestação livre e espontânea dos clientes para com os diversos tipos de pesquisas e tratamentos aos quais estavam sendo sujeitos.

\section{Resultados da avaliação}

Os resultados da aplicação dos questionários demonstraram também que a identificação do componente somático, emocional ou miscelânea da dor é de fundamental importância para o sucesso do tratamento e a reunião entre bolsistas e coordenador deixou isso muito claro.

O questionário foi aplicado antes dos pacientes iniciarem seus tratamentos na FORP-USP e após um ano. Foram entrevistados 39 pacientes (sete homens e 32 mulheres), com idades que variaram de dezesseis a 65 anos. A pontuação do Questionário McGill pode ser analisada de acordo com o número de descritores escolhidos (análise quantitativa) e/ou em relação à pontuação atrelada a estes descritores (análise qualitativa) [3]. Neste trabalho foram aplicadas as duas formas de análise.

Neste contexto, observamos que os pacientes na avaliação escolheram de cinco a vinte (média de quinze) termos para descrever a dor e a dimensão afetiva/avaliativa, e com exceção de um paciente, a dor sempre foi pontuada. As outras dimensões tiveram uma média de termos escolhidos de oito para sensorial, três para afetiva, um para avaliativa e dois para mista sensorial (ver Anexo, Tabelas 1 e 2). 


\section{Resultados da reavaliação}

Do total de pessoas a que aplicamos o questionário, dezesseis responderam a reavaliação. Ao reavaliarmos estes pacientes, eles escolheram de zero a vinte (média de doze) termos para descrever a dor e a dimensão afetiva/avaliativa. As outras dimensões tiveram uma média de termos escolhidos de seis para sensorial, dois para afetiva, um para avaliativa e um para mista sensorial (ver Anexo, Tabelas 3 e 4).

A avaliação quantitativa da sintomatologia dolorosa foi realizada no tempo inicial (após a primeira aplicação do McGill Pain Questionnaire [5] e aplicação da Escala Visual Analógica-EVA). Dias depois, os pacientes receberam placas miorrelaxantes para alívio e tratamento da sintomatologia dolorosa. Os pacientes foram acompanhados por três anos, sendo que neste período foram substituídas as placas conforme necessário. Os resultados das avaliações quantitativas da sintomatologia dolorosa antes da avaliação e na reavaliação são apresentados nos Gráficos 1 e 2 (ver Anexo).

Quanto ao questionamento referente a sintomas que acompanham a patogenia (dor), o relato de dor de cabeça manteve-se inalterado (38\%) sendo que o relato de tontura apresentou leve alteração $(22 \%)$. Com relação à sonolência, nota-se melhora significativa, visto que passou de $13 \%$ para $4 \%$ de relatos.

Em relação aos itens ao fim do questionário (Figura 1) que são descritos como "sintomas que acompanham", "sono", "ingestão de alimentos" e "atividades", os resultados serão apresentados em Gráficos descritivos de porcentagem (ver Anexo, Gráficos 2 a 5 ).

Para a avaliação do sono, pode-se considerar que houve importante melhora, visto que não houve relatos de insônia na reavaliação. Na avaliação, $24 \%$ dos pacientes referiram insônia devido à sintomatologia dolorosa. Ao questionarmos sobre a ingestão de alimentos na reavaliação, a maioria (41\%) referiu ter boa alimentação, sendo que na avaliação, apenas 29\% apresentaram esse relato. Quanto à realização de atividades, $56 \%$ dos pacientes referiram manter boa quantidade de atividades na reavaliação, sendo que na avaliação apenas $31 \%$ relataram ter boa atividade.

\section{DISCUSSÃO}

As atividades propostas foram atendidas nesse projeto em todas as dimensões previstas e pactuadas. Após a autorização das atividades clínicas/entrevistadoras pelas bolsistas e já com assinatura do termo de consentimento livre e esclarecido do supervisor de clínicas e dos responsáveis pelas disciplinas clínicas da FORP-USP, os estudantes de graduação envolvidos no projeto selecionaram os clientes (pacientes inscritos via convênio SUS com a disciplina de oclusão da FORP - 8041203) que preenchiam os requisitos para ingressar no projeto: portadores de DTMs crônicas, em tratamento nas clínicas da FORP, sendo considerados de ambos os gêneros e com idade acima de dezesseis anos.

As bolsistas, de posse das fichas clínicas, e após conhecimento detalhado dos casos, iniciaram o processo de entrevistas onde o cliente teve toda liberdade de expressar seus problemas relacionados à dor, sendo investigada a história médica anterior, familiar, história dental, profissão, uso de medicamentos etc. [4,5]. 
Foi então aplicada a versão em português do McGill Pain Questionnaire [5], para diferenciação entre dor somática, afetiva ou miscelânea, e uma Escala Visual Analógica (EVA) para mensuração da dor a cada cliente que foi incluído no projeto. Os resultados foram tabulados e discutidos primeiramente pelo coordenador do projeto e estudantes envolvidos, e em uma segunda etapa, com os docentes clínicos onde o projeto foi desenvolvido.

O sucesso do projeto ficou evidenciado quando, por iniciativa das bolsistas, uma simples pergunta foi adicionada ao relatório: "De todos os questionários e entrevistas que o Sr. (Sra.) foi solicitado responder até o presente momento, teve alguma pergunta que gostaria que fosse dirigida a você e que não foi realizada?"

As respostas foram quase que unânimes e a pergunta a ser feita era: "Você está satisfeito (a) com o atendimento e suas queixas foram ouvidas?”

O cômputo de dados clínicos e explanatórios da parte das bolsistas e dos clientes levou-nos a refletir que o cliente nem sempre é ouvido sobre o que o levou a procurar o serviço de atendimento em saúde, e que muitas vezes há uma descrença sobre a preocupação de nós, clínicos, com relação aos nossos clientes. Assim, graduação e pesquisa ficam atendidas, mas o oferecimento do produto (tratamento) é falho na interlocução entre a academia e a sociedade, o que corrobora com dados de Sawada e colaboradores [4].

Foi observada a necessidade de acrescentar à parte inicial do questionário tópicos como idade, profissão, e questões como "Existe algo que provoca a sua dor?", "Há quanto tempo você sente esta dor?”, "Adaptou-se ao uso da placa? Sentiu alívio da sua dor?", para melhor análise do quadro do paciente.

O maior problema enfrentado foi relativo à autorização de alguns docentes para a participação das bolsistas na aplicação dos questionários. Das três disciplinas na graduação e na extensão que foram procuradas, somente uma da graduação ofereceu a clínica e toda liberdade de atuação das bolsistas.

Uma das bolsistas pertence ao Curso de Graduação em Fisioterapia da Faculdade de Medicina de Ribeirão Preto da USP, e um (pequeno) problema se instalou quando uma docente levantou a possibilidade de uma estudante da graduação de outra unidade atuar em nossas clínicas sem seguro-saúde. Numa breve reunião estabelecemos que a bolsista iria apenas aplicar o questionário, acompanhada, ainda, da outra bolsista da graduação da Odontologia, e tudo foi resolvido de forma amplamente satisfatória.

Nota-se ainda que palavras como interdisciplinaridade, transdisciplinaridade, multidisciplinaridade etc., cada vez mais correntes em processos didático-pedagógicos, ainda restringem-se ao "pensar", "refletir", "vislumbrar”, e estão muito pouco presentes no "agir", o que está em direção oposta à situação ideal, que seria a apresentada por Sawada e colaboradores [4].

Inicialmente, outra dificuldade que sentimos foi em conseguir a autorização do Comitê de Ética em Pesquisa da FORP para a realização do nosso projeto devido à formatação do mesmo não obedecer estritamente os moldes e ditames da pesquisa clínica pura. Mas bastou uma orientação na forma de ofício oferecida pelo coordenador do projeto para o Comitê, informando que os objetivos eram, sim, de ensino e pesquisa, mas atrelados às atividades de extensão, onde os objetivos primários não são "apenas" de ensino puro ou de pesquisa pura, mas de realmente aplicar a indissociabilidade das atividades fins da universidade na interlocução com a sociedade. 
O agendamento para reavaliação após o período de três a doze meses de tratamento foi mais um empecilho ao curso do projeto, visto que muitos pacientes já haviam recebido alta e não pretendiam retornar à instituição, também devido à mudança dos números telefônicos, ou ainda pela dificuldade de transporte dos pacientes.

Ao observar os valores atribuídos às pontuações dos descritores percebe-se que a gravidade da queixa diminuiu na contagem qualitativa dos descritores de dor (ver Anexo, Gráfico 2), mostrando que pode existir uma correlação deste resultado com o fato de o tratamento recebido por estes pacientes ter sido quase que majoritariamente odontológico, o que destaca a necessidade para estes pacientes de um tratamento multidisciplinar que englobe suas necessidades biopsicossociais. Talvez dessa forma eles tivessem um percentual maior de melhora nos descritores. Outro aspecto a ser analisado é o costume errôneo dos pacientes de abandonar o tratamento assim que a dor cessa ou diminui, tornando, dessa forma, seu estado de bem-estar não mais duradouro e susceptível à volta dos sintomas de dor.

Sugere-se, em trabalhos futuros, também a análise do impacto da dor no cotidiano do paciente, porque às vezes o quadro de dor permanece, porém o indivíduo torna-se capaz de realizar mais atividades ou de realizar as mesmas de antes, mas com maior qualidade, pois a dor não o incomoda tanto. Para tanto, pode-se explorar mais o tópico "Atividades" do próprio questionário McGill.

As sugestões descritas objetivam aumentar a qualidade técnica do atendimento multidisciplinar em saúde e propiciar uma avaliação mais fidedigna e completa do paciente, favorecendo um melhor atendimento e tratamento de sua queixa. Cumpre salientar que dentre os pacientes reavaliados somente um realizou acompanhamento odontológico e fisioterapêutico. Quando os pacientes foram orientados quanto ao papel da fisioterapia complementando o tratamento odontológico, todos demonstraram interesse em realizá-lo e esta vontade foi expressa por meio da solicitação de encaminhamento ao serviço de fisioterapia oferecido na Clínica de Pacientes Especiais.

\section{CONCLUSÕES}

Após três anos de acompanhamento clínico de 39 pacientes, com avaliação da dor quantitativa e qualitativamente, pode-se concluir que as expectativas dos clientes e cuidadores com relação ao tratamento clínico das DTMs nem sempre são atendidas em igual importância, apesar de o tratamento clínico com a placa miorrelaxante, no caso, ter sido satisfatório. Notou-se ainda a falta de adesão ao tratamento e seguimento de caso após o cessamento da dor, o que pode implicar um recrudescimento do problema em médio e longo prazo. Nem sempre o olhar do profissional de saúde é coincidente com a necessidade do cliente, $o$ que demonstra clara necessidade de interdisciplinaridade e melhor diálogo entre as partes.

\section{REFERÊNCIAS BIBLIOGRÁFICAS}

[1] CHAPMAN, C. R. Pain measurement: an overview. Pain, v. 22, n. 1, pp. 1-31, 1985. 
[2] HAAS, D. A. Pharmacologic considerations in the management of temporomandibular disorders. Journal of the Canadian Dental Association, v. 61, n. 2, pp. 105-109, 112-114, 1995.

[3] MARTINEZ, J. E.; GRASSI, D. C.; MARQUES, L. G. Análise da aplicabilidade de três instrumentos de avaliação de dor em distintas unidades de atendimento: ambulatório, enfermaria e urgência. Revista Brasileira de Reumatologia, v. 51, n. 4, pp. 299-308, 2011.

[4] SAWADA, N. O.; PEDRAZZI, V.; RODRIGUES, M. L. V. $1^{\text {st }}$ Forum on integration of health services with USP teaching/learning, research and community activities. Revista Cultura e Extensão USP, v. 6, pp. 33-40, 2012.

[5] VAROLI, F. K.; PEDRAZZI, V. Adapted Version of the MPQ to Brazilian Portuguese. Brazilian Dental Journal, v. 17, pp. 328-335, 2006.

BÁRBARA GRAZIELE RAMOS graduanda em Fisioterapia da Faculdade de Medicina de Ribeirão Preto da Universidade de São Paulo.

SAMIRA FALLEIROS ORTIZ graduanda em Odontologia da Faculdade de Odontologia de Ribeirão Preto da Universidade de São Paulo.

TAMIRIS BITTENCOURT FONSECA graduanda em Fonoaudiologia da Faculdade de Medicina de Ribeirão Preto da Universidade de São Paulo.

VINICIUS PEDRAZZI professor associado do Departamento de Materiais Dentários e Prótese da Faculdade de Odontologia de Ribeirão Preto da Universidade de São Paulo - Av. do Cafés/n - CEP 14040904 - Ribeirão Preto-SP - tel.: (16) 3602-4008 - fax: (16) 3602-0547 - e-mail: pedrazzi@forp.usp.br. 
ANEXO

Tabela 1 Número de descritores escolhidos na avaliação

\begin{tabular}{|c|c|c|}
\hline & SEXO FEMININO & SEXO MASCULINO \\
\hline Número de descritores & 32 & 7 \\
\hline Idade & 37,5 & 44 \\
\hline Sensorial & 7,8 & 8 \\
\hline Afetiva & 2,8 & 2,4 \\
\hline Avaliativa & 1 & 1 \\
\hline Mista sensorial & 2 & 1,9 \\
\hline Mista afetiva/ avaliativa & 0,9 & 1 \\
\hline Mista total & 3 & 2,9 \\
\hline Escore total & 16 & 15 \\
\hline
\end{tabular}

Tabela 2 Média de pontuação referente aos descritores escolhidos na avaliação por gênero

\begin{tabular}{lcc}
\hline & SEXO FEMININO & SEXO MASCULINO \\
\hline Idade & 37,5 & 44 \\
\hline Sensorial & 22,53 & 22,87 \\
\hline Afetiva & 5,84 & 4,57 \\
\hline Avaliativa & 3,12 & 2,6 \\
\hline Mista sensorial & 4,5 & 3 \\
\hline Mista afetiva/ avaliativa & 3,18 & 3,88 \\
\hline Mista total & 7,68 & 7,28 \\
\hline Escore total & 39,15 & 36,57 \\
\hline
\end{tabular}

Tabela 3 Número de descritores escolhidos na reavaliação

\begin{tabular}{|c|c|c|}
\hline & SEXO FEMININO & SEXO MASCULINO \\
\hline Idade & 38 & 43,5 \\
\hline Sensorial & 6,9 & 2,6 \\
\hline Afetiva & 3,4 & 3,3 \\
\hline Avaliativa & 0,9 & 1 \\
\hline Mista sensorial & 2 & 1,8 \\
\hline
\end{tabular}




\begin{tabular}{lcc}
\hline & SEXO FEMININO & SEXO MASCULINO \\
\hline Mista afetiva/ avaliativa & 0,8 & 1 \\
\hline Mista total & 2,8 & 2,8 \\
\hline Escore total & 15 & 15 \\
\hline
\end{tabular}

Tabela 4 Pontuação referente aos descritores escolhidos na reavaliação

\begin{tabular}{|c|c|c|}
\hline & SEXO FEMININO & SEXO MASCULINO \\
\hline Idade & 38 & 43,5 \\
\hline Sensorial & 19,8 & 19,7 \\
\hline Afetiva & 5,1 & 7 \\
\hline Avaliativa & 2,6 & 3 \\
\hline Mista sensorial & 4,7 & 3,7 \\
\hline Mista afetiva/ avaliativa & 2,3 & 3,1 \\
\hline Mista total & 7 & 7,3 \\
\hline Escore total & 33,6 & 35 \\
\hline
\end{tabular}

Gráfico 1 Análise quantitativa Índice atual da dor (EVA) - primeira avaliação

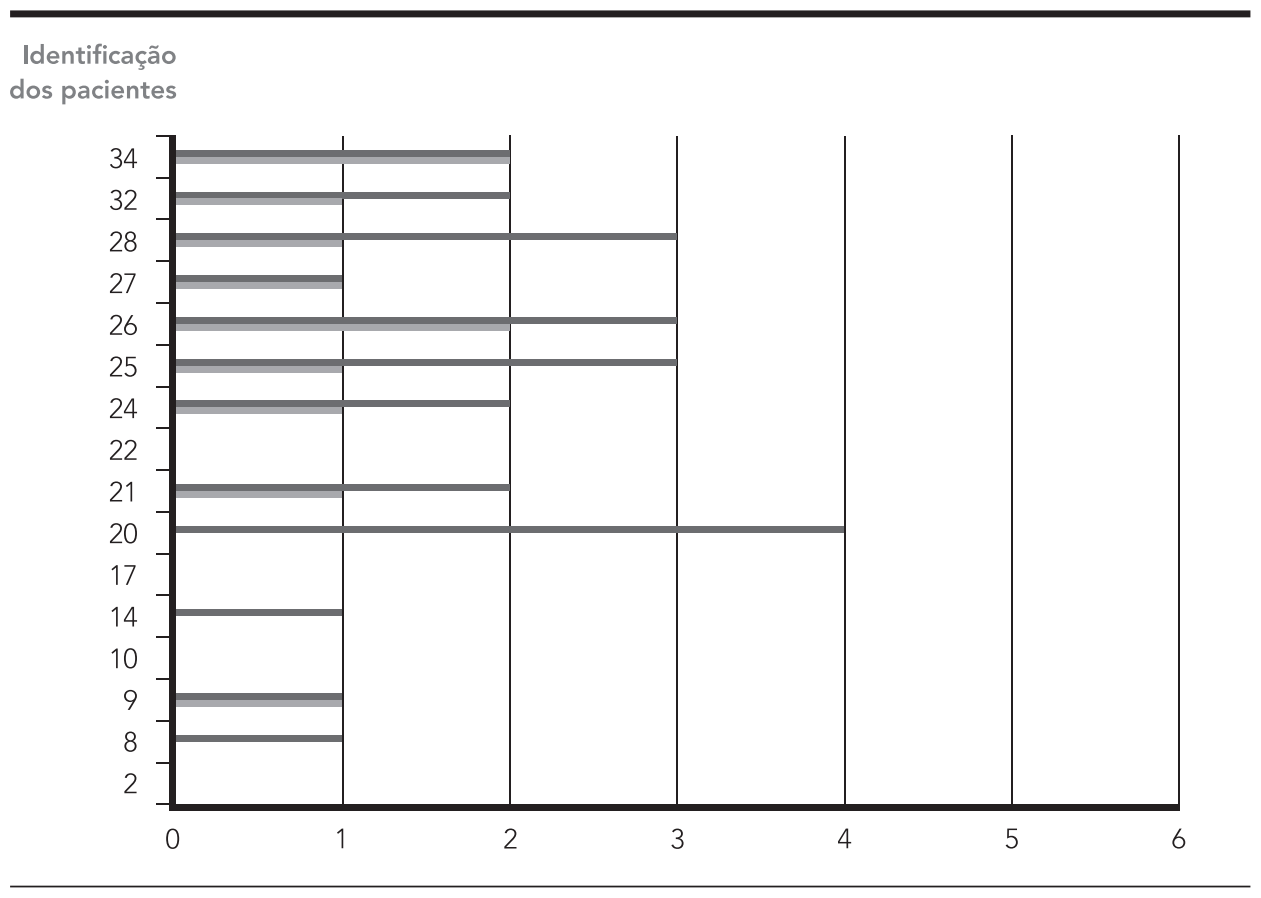

Inicial

$\square$ Final 
Gráfico 2 Análise qualitativa Índice atual da dor (EVA) - reavaliação

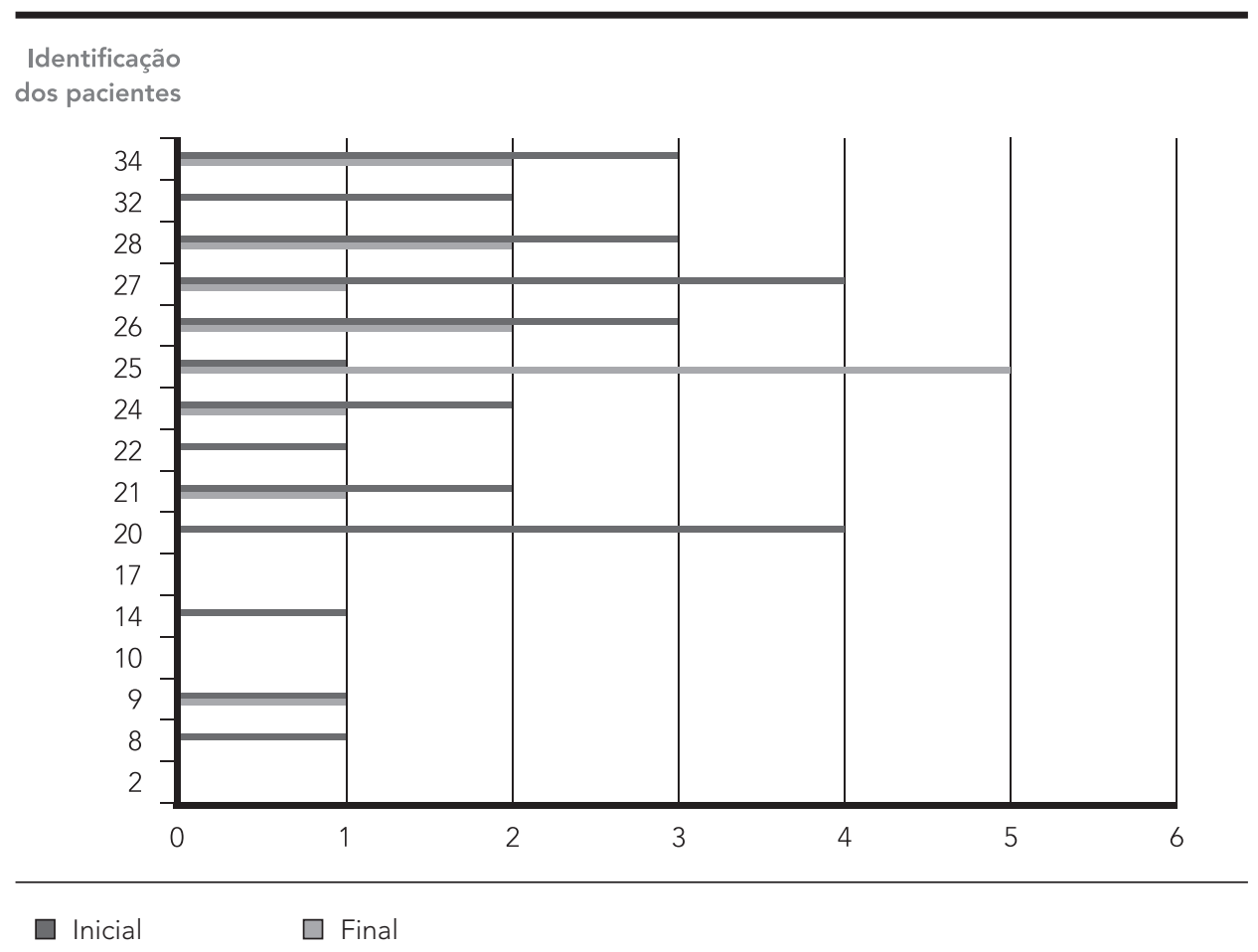

Gráfico 3 Sintomas que acompanham a patogenia (antes e depois do tratamento)

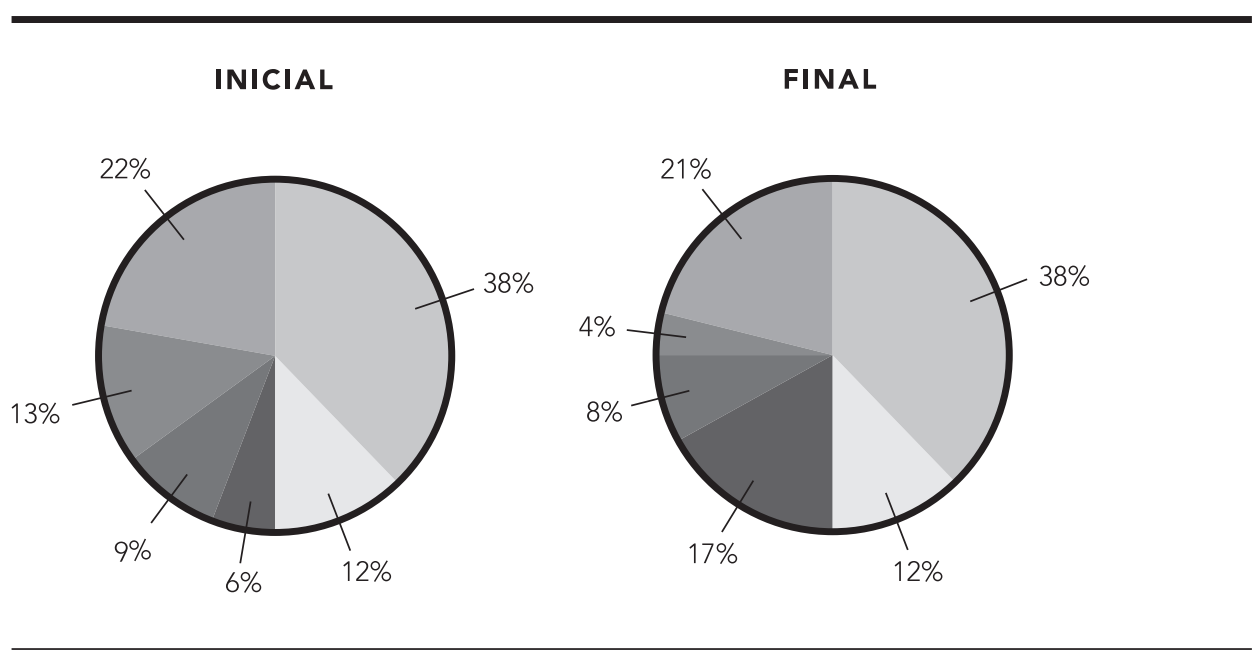
$\square$ Náusea
$\square$ Dor de Cabeça
$\square$ Tontura
$\square$ Sonolência
- Constipação
Diarréia 
Gráfico 4 Avaliação da qualidade do sono

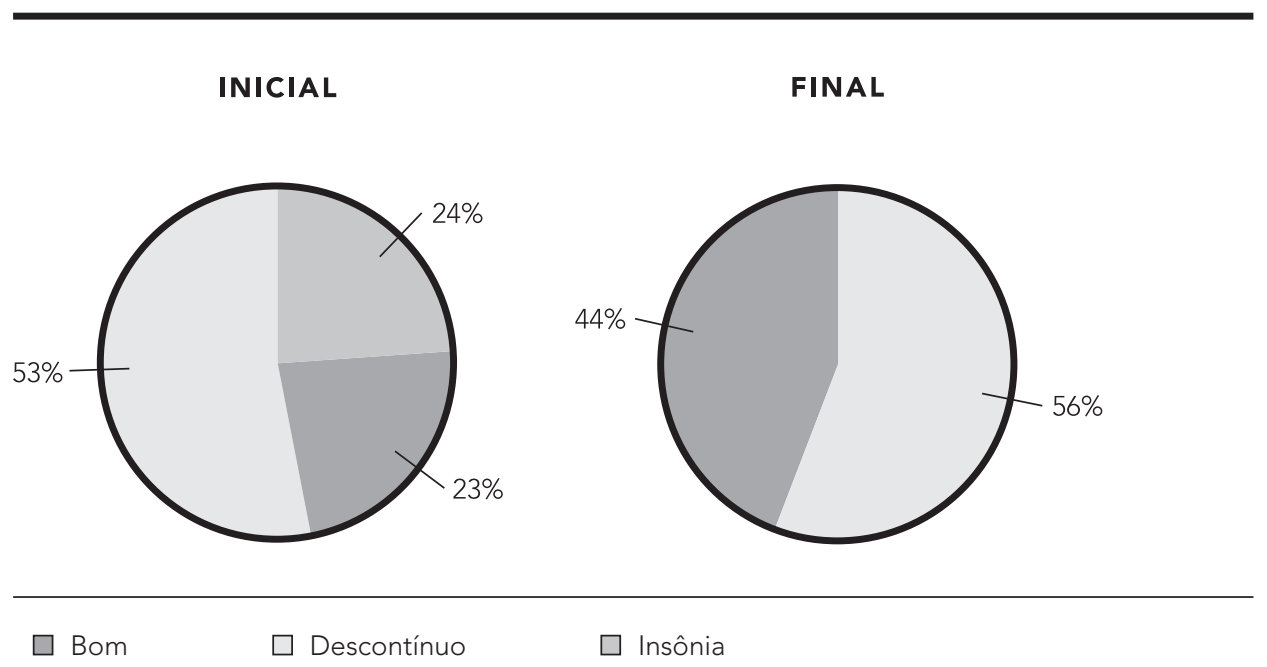

Gráfico 5 Avaliação da ingestão de alimentos

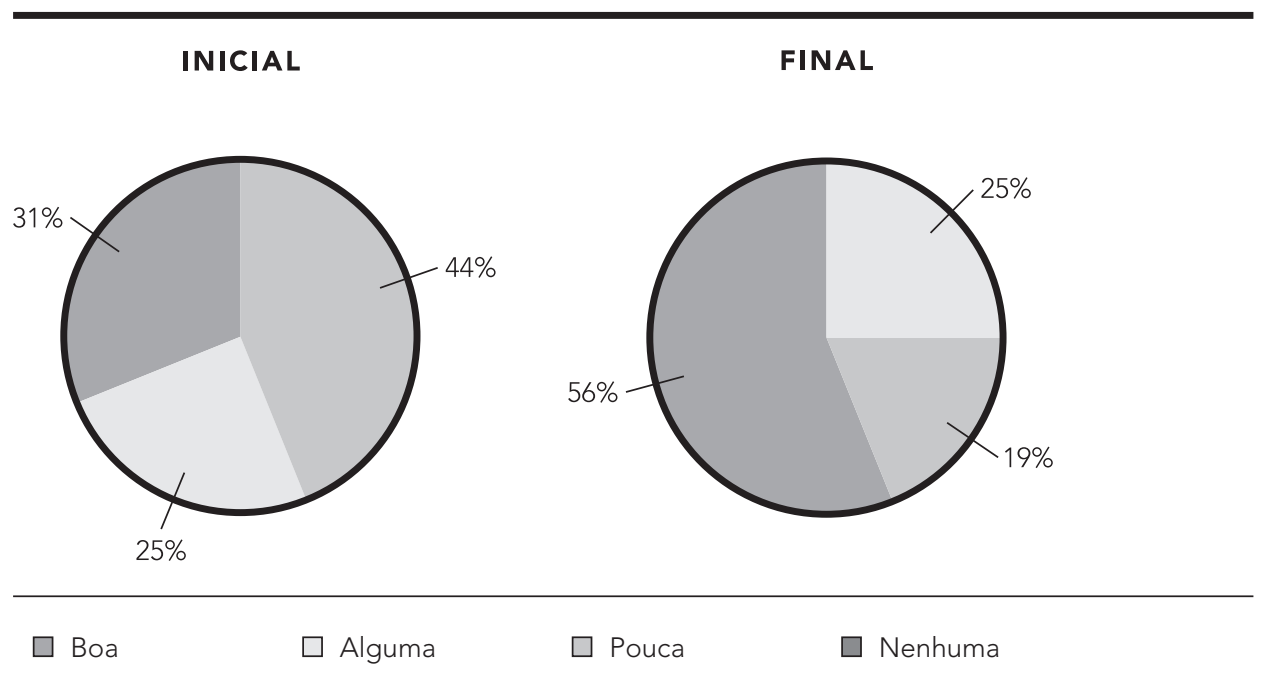


Gráfico 6 Avaliação das atividades desenvolvidas em função da presença de dor

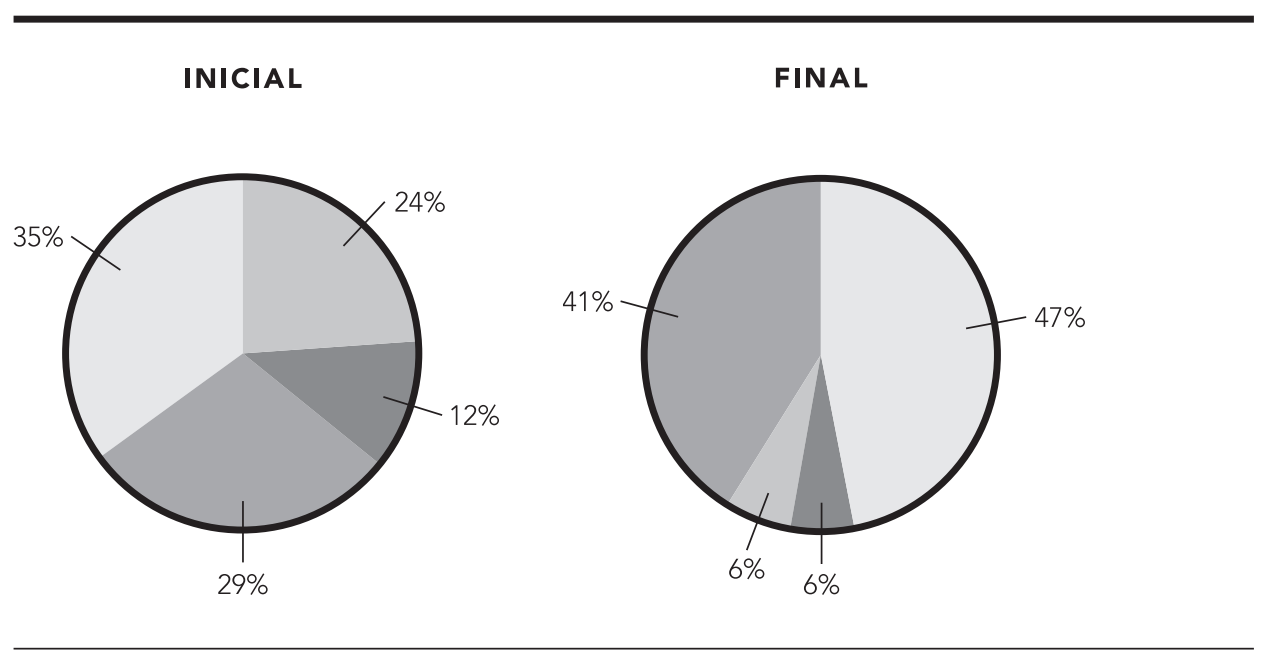

$\square$ Boa $\square$ Alguma $\quad \square$ Pouca $\quad \square$ Nenhuma 